\title{
AVALIAÇÃO DO RISCO GERENCIAL NA ELABORAÇÃO DE PROJETOS DE INOVAÇÃO EM PEQUENAS E MÉDIAS EMPRESAS DE ALTA TECNOLOGIA SOB A ÓTICA DA METODOLOGIA PRISM.
}

\section{Taciana de Barros Jerônimo}

Mestre em Engenharia de Produção pela Universidade Federal de Pernambuco - UFPE E-mail: taciana.barros@gmail.com (Brasil)

\section{Lúcio Camara e Silva}

Mestre em Engenharia de Produção pela Universidade Federal de Pernambuco - UFPE E-mail: luciocsilva@gmail.com (Brasil)

\section{Deise de Araújo Batista}

Mestre em Engenharia de Produção pela Universidade Federal de Pernambuco - UFPE Pesquisadora em Engenharia de Produção pela Universidade Federal de Pernambuco - UFPE E-mail: deisedab@gmail.com (Brasil)

\section{Marcella Brito Galvão}

Mestre em Engenharia de Produção pela Universidade Federal de Pernambuco - UFPE Pesquisadora em Engenharia de Produção pela Universidade Federal de Pernambuco - UFPE E-mail: marcella_brito@yahoo.com.br (Brasil) 


\title{
AVALIAÇÃO DO RISCO GERENCIAL NA ELABORAÇÃO DE PROJETOS DE INOVAÇÃO EM PEQUENAS E MÉDIAS EMPRESAS DE ALTA TECNOLOGIA SOB A ÓTICA DA METODOLOGIA PRISM.
}

\section{RESUMO}

Este estudo de caso tem o objetivo de analisar as falhas da atividade de gerenciamento de projetos em pequenas e médias empresas de alta tecnologia, utilizando a ferramenta PRISM. Essa metodologia, proposta por Gary Pan, Ray Hackney e Shan L. Pan, analisa as interações post mortem entre os componentes do projeto durante o curso de sua execução como uma relação de intercâmbio entre a organização do projeto, o sistema de informação e os seus usuários. Assim, a contribuição desse trabalho é permitir uma análise formal das relações entre a sucessão de eventos de desenvolvimento de projetos de cunho inovativo e fornecer insights sobre o sucesso potencial ou o fracasso em projetos de pequenas e médias empresas de alta tecnologia (PMET).

Palavras-chaves: Projeto; Pequena e Média Empresa; Análise de Falhas.

\section{RISK MANAGEMENT PROJECTS EVALUATION OF INNOVATION IN SMALL AND MEDIUM-HIGH TECHNOLOGY USING THE PERSPECTIVE OF PRISM METHODOLOGY}

\begin{abstract}
This study aims to analyze the failures of the activity of project management in small and mediumsized high-tech firms, using the tool PRISM. This postmortem methodology, proposed by Gary Pan, Shan L. and Ray Hackney Pan, analyzes the interactions between the components of the project during the exchange relationship between the project organization, information system and the users. Thus, the contribution of this work is to allow a formal analysis of the relationship between the sequence of events development projects into an innovative view, that provide insights into the potential success or failure of the project on medium-sized high-tech firms (whose the acronym is PMET).
\end{abstract}

Keywords: Project; Small and Medium Enterprise; Failure Analysis. 


\section{INTRODUÇÃo}

$\mathrm{Na}$ maioria dos casos, os objetivos operacionais originais dos projetos de tecnologia da informação (TI) não são concretizados, mesmo com investimentos de somas financeiras consideráveis.

O fantasma do fracasso dos sistemas da informação assombra as organizações, uma vez que a taxa de erro nos projetos finais é de 70\% (Drummond, 2005). Esse índice refere-se à incapacidade de lidar com a complexidade dos sistemas. De modo geral, as falhas em TI são devidas aos fatores organizacionais e sociais e aos elementos técnicos de desenvolvimento.

Os projetos são empreendimentos independentes, temporários com propósito e objetivo distintos e definidos, possuem recursos e administração próprios. São divididos de acordo com os componentes de seu ciclo de maturação e apresentam-se em cinco grupos, a saber: iniciação, planejamento, execução, monitoramento/controle e encerramento (Project Management Body of Knowledge [PMBOK], 2004). Para fins de gerenciamento, cada um é decomposto em nove áreas de conhecimento a saber: integração, escopo, tempo, custos, qualidade, recursos humanos, comunicações, riscos e aquisições.

A constituição dos projetos nessas nove áreas visa atender à aplicação dos conhecimentos, das habilidades e das técnicas realizadas em seus processos e atividades, as quais devem satisfazer as necessidades e expectativas das partes envolvidas. Essas áreas foram descritas no PMBOK, guia de gerenciamento de projetos (GP) e base metodológica do Project Management Institute (PMI). Esse último é baseado em processos, descreve de forma organizada como o trabalho e os eventos dos projetos devem ser elaborados.

O risco é considerado como uma das nove áreas de conhecimento do projeto e pode ser entendido como um evento ou condição incerta em pelo menos um de seus objetivos, em qualquer fase de sua execução.

O gerenciamento de riscos de um projeto inclui os processos que tratam da identificação, monitoramento, controle e planejamento dos eventos, com o objetivo de eliminar ou mitigar a probabilidade e o impacto dos eventos negativos e potencializar os positivos. Contudo, a decisão em relação ao risco vai depender da atitude de cada gestor e sua capacidade de suportá-lo.

Nas empresas de alta tecnologia o risco nos projetos inovativos é alto. Segundo a pesquisa PINTEC no ano de 2005, os principais obstáculos para inovação apontados pelos empresários, são: elevados custos de investimento inicial em pesquisa e desenvolvimento (P\&D), riscos econômicos excessivos ao retorno do capital investido e escassez de fontes de financiamento (IBGE, 2005). 
Destaca-se na área de informática que as atividades internas de P\&D (2,33\%), a aquisição de máquinas e equipamentos $(1,27 \%)$ e o treinamento $(0,69 \%)$ respondem pelas três maiores parcelas dos gastos com inovação no total da receita líquida de vendas (IBGE, 2005). No ano de 2006 esse setor era composto por 51.240 empresas com 34.4043 pessoas empregadas e R $\$$ 8,3 bilhões em pagamento de salários, retiradas e outras remunerações (IBGE, 2006).

\subsection{ESTRUTURAÇÃO DO TRABALHO}

A realização desse trabalho foi motivada pela escassez de estudo de técnicas e ferramentas específicas de gerenciamento de riscos em projetos de pequena e média empresa de informática cujo foco é o desenvolvimento de tecnologia de ponta.

Sabe-se que as atividades de GP interligam-se pelos resultados, desse modo, o objetivo principal do estudo foi analisar o desenvolvimento dos processos de gerenciamento de projetos de cunho inovativo, com a utilização da ferramenta PRISM, proposta por Gary Pan, Ray Hackney e Shan L. Pan (2008), a qual demonstra condições antecedentes, eventos críticos e resultados ao longo do tempo de execução do projeto em uma relação de intercâmbio para sua trajetória postmortem.

Os eventos críticos serão correlacionados aos seguintes objetivos de desempenho: flexibilidade, custo, credibilidade, rapidez e qualidade. A metodologia PRISM analisa as interações cíclicas entre os componentes do projeto durante o curso da execução, em cada evento crítico.

Assim, esse trabalho apresenta-se organizado da seguinte maneira: no item 2 estuda-se as empresas de alta tecnologia e os riscos dos projetos desenvolvidos por elas; no item 3 é conceituada a metodologia para a elaboração do protótipo da ferramenta utilizada; no item 4 é apresentada a empresa estudo de caso; no item 5 são relatados os resultados coletados e aferidos; e, por último, no item 6, são relatadas as considerações finais.

Revista de Gestão e Projetos - GeP, São Paulo, v. 2, n. 1, p 52-68, jan./jun. 2011. 


\section{EMPRESAS DE ALTA TECNOLOGIA}

De acordo com Marcovitch et al. (1986) "empresas de alta tecnologia são entes jurídicos criados para fabricar produtos ou serviços que utilizam alto conteúdo tecnológico, dispõem de competência rara ou exclusiva em termos de produtos ou processos, viáveis comercialmente, que incorporam grau elevado de conhecimento científico".

Utiliza de mão-de-obra altamente especializada e há a adoção sistemática de inovação tecnológica em produto e em processo. Essas são características de empresas de tecnologia de ponta, de acordo com Fonseca e Kruglianskas (2002). Essas organizações podem ainda ser conceituadas a partir do dispêndio em atividades de P\&D em consonância ao domínio de alta intensidade tecnológica, segundo a Organisation for Economic Co-operation and Development (OECD) (2000), são os setores: aeroespacial, farmacêutico, informática, eletrônica e telecomunicações e de instrumentos.

\subsection{PROJETOS DE ALTA TECNOLOGIA}

A própria geração de "tecnologia se desenvolve por meio da transferência dos conhecimentos acumulados em práticas organizacionais" (Campos e Barbieri, 2002) pelas empresas de tecnologia de ponta.

Nesse sentido, para Jerônimo et al. (2009) a inovação envolve uma teia extremamente entrelaçada de interações na busca pela competitividade das pequenas e médias empresas, cujos elementos norteadores para o estabelecimento da vantagem competitiva de desenvolvimento de um projeto, são:

- Origem das empresas em nichos de mercado não englobados por grandes empresas;

- Estrutura flexível, maior disponibilidade dos fluxos de informação;

- Espírito empreendedor dos líderes, o qual gera sinergia entre os funcionários, fornecedores e clientes;

- Troca de conhecimento a partir de mecanismos informais de aprendizado e do relacionamento entre os funcionários;

- Isenção ou redução de tributos; 
- Grandes empresas, desenvolvedoras de software e hardware apoiam esse porte empresarial, ao promoverem treinamento e desconto ou redução nos preços das licenças de uso da tecnologia.

Essa configuração exige uma estrutura organizacional orgânica, a qual possa manter os fluxos de informação entre os funcionários e a troca de conhecimento para melhoria da qualidade dos projetos inovativos (Murphy e Ledwith, 2007). De acordo com Freire (2000), o ciclo de desenvolvimento de um projeto é constituído por seis fases influenciadas de acordo com o porte da empresa, conforme a Tabela 1.

Tabela 1- Gestão do ciclo da inovação.

\begin{tabular}{|c|c|c|c|}
\hline $\begin{array}{l}\text { FASE DO CICLO } \\
\text { (PMBOK) }\end{array}$ & $\begin{array}{l}\text { FASE DO CICLO } \\
\text { (INOVAÇÃO) }\end{array}$ & MÉDIAS EMPRESAS & PEQUENAS EMPRESAS \\
\hline Iniciação & $\begin{array}{l}\text { Identificação da } \\
\text { oportunidade }\end{array}$ & $\begin{array}{c}\text { Análise informal e } \\
\text { qualitativa do mercado }\end{array}$ & $\begin{array}{l}\text { Conversas com clientes e } \\
\text { análise da concorrência }\end{array}$ \\
\hline Planejamento & Geração da ideia & $\begin{array}{l}\text { Sessões de reuniões e } \\
\text { questionamento aos } \\
\text { clientes }\end{array}$ & $\begin{array}{l}\text { Questionamento aos clientes } \\
\text { e benchmarking das } \\
\text { empresas líderes }\end{array}$ \\
\hline Execução & Teste da inovação & $\begin{array}{c}\text { Inovação e análise do } \\
\text { mercado }\end{array}$ & $\begin{array}{c}\text { Inovação e aprendizagem } \\
\text { pelos erros }\end{array}$ \\
\hline Monitoramento & $\begin{array}{l}\text { Introdução da } \\
\text { inovação }\end{array}$ & $\begin{array}{c}\text { Impacto médio e análise } \\
\text { dos clientes }\end{array}$ & $\begin{array}{l}\text { Baixo impacto e análise de } \\
\text { alguns clientes }\end{array}$ \\
\hline Encerramento & $\begin{array}{l}\text { Gestão da difusão da } \\
\text { inovação }\end{array}$ & Gestão reativa da inovação & $\begin{array}{l}\text { Gestão oportunista da } \\
\text { inovação }\end{array}$ \\
\hline
\end{tabular}

Fonte: Adaptado de Freire (2000).

Assim, pode-se dizer que a análise das falhas de projetos tecnológicos em cada fase do ciclo apresentada na Tabela 1, auxilia a melhoria dos resultados em fatores como treinamento, compromisso profissional, conhecimento de ferramentas técnicas, visão sistêmica e apoio da alta administração (Carvalho e Rabechini, 2008).

Revista de Gestão e Projetos - GeP, São Paulo, v. 2, n. 1, p 52-68, jan./jun. 2011. 


\subsection{ANÁliSE DO RISCO DOS PROJETOS DE ALTA TECNOLOGIA}

Decisões que envolvem tecnologia nunca são pontuais e normalmente implicam em alto custo e, por isso, requerem extraordinários cuidados para a redução de riscos (Vendrametto, 2007). Elas envolvem necessidade, aprendizagem, riscos e custos que são influenciadas por diversos fatores de natureza diversa, dentre eles se destaca a escala econômica nacional e a externa.

Quando a incerteza é alta, o processo de desenvolvimento necessita de flexibilidade para lidar com as mudanças. Segundo Turner et al.(2009) os projetos com alta incerteza são especialmente difíceis de definir e de estabelecer objetivos realísticos especificados. A especificação consiste em uma descrição minuciosa de um produto contendo os requisitos que definem sua função e outras características. A maior dificuldade em desenvolver um novo processo ou produto é a ausência de dados que auxiliem a descrição das características (Castellano, 1996). Outros fatores de risco aos empreendimentos das organizações de alta tecnologia estão dispostos a seguir:

- Fatores políticos - apesar da criação de leis que incentivam o desenvolvimento da ciência e tecnologia, as quais promovem redução de impostos e taxas, muitas empresas de alta tecnologia ficam marginalizadas por não terem acesso aos benefícios.

- Fatores sociais - a camada mais jovem de indivíduos que tiveram acesso ao ensino superior é contratada. Muitos deles constituíram grupos de trabalho dentro das faculdades e universidades e ao se graduarem formalizam uma empresa, fenômeno conhecido por spinoff. Contudo, não têm capacidade para administrar o próprio empreendimento. Outros elementos estão associados aos custos de reter o profissional com alto know-how, aos dispêndios de treinamento e aperfeiçoamento profissional e do elevado turn-over dos colaboradores.

- Fatores mercadológicos - os proprietários geralmente não conhecem o potencial do mercado, o que provoca ausência de credibilidade e de estratégias para colocar os primeiros produtos elaborados no mercado.

- Fatores econômicos - os próprios empresários empreendem o negócio sem estudo e planejamento acerca das ameaças do mercado, sucessivamente desenvolvem inovações com recursos provenientes de terceiros. Há escassez na disponibilidade de recursos nas instituições de crédito privadas ou públicas.

Revista de Gestão e Projetos - GeP, São Paulo, v. 2, n. 1, p 52-68, jan./jun. 2011. 


\section{A METODOLOGIA}

A metodologia de estudo de caso adotado nesse trabalho tem abordagem quantitativa. $\mathrm{Na}$ qual os projetos concluídos de uma empresa de informática foram analisados para a construção do modelo. Para a coleta de dados foram utilizados questionários, aplicados aos líderes da empresa do estudo.

Para a pesquisa foram entrevistados 4 líderes e analisados 10 projetos da empresa do estudo. A coleta de dados abrangeu o período de março de 2009 a março de 2010.

A edição dos dados foi elaborada pela análise post-mortem (APM) proposta por Pan et al. (2008) com o objetivo de captar as experiências por intermédio de entrevistas em projetos já formalizados e de disseminar as metas de desempenho ou fracasso aos demais projetos, para a empresa aprender com seus erros e acertos.

Os projetos foram julgados com base nos cinco objetivos de desempenho: qualidade, rapidez, credibilidade, flexibilidade e custo. O modelo de relações de intercâmbio, proposto por Sauer (1993), é muito importante para o entendimento do artigo, o qual pode ser analisado na Figura 1.

Figura 1- Modelo de intercâmbio de relações.

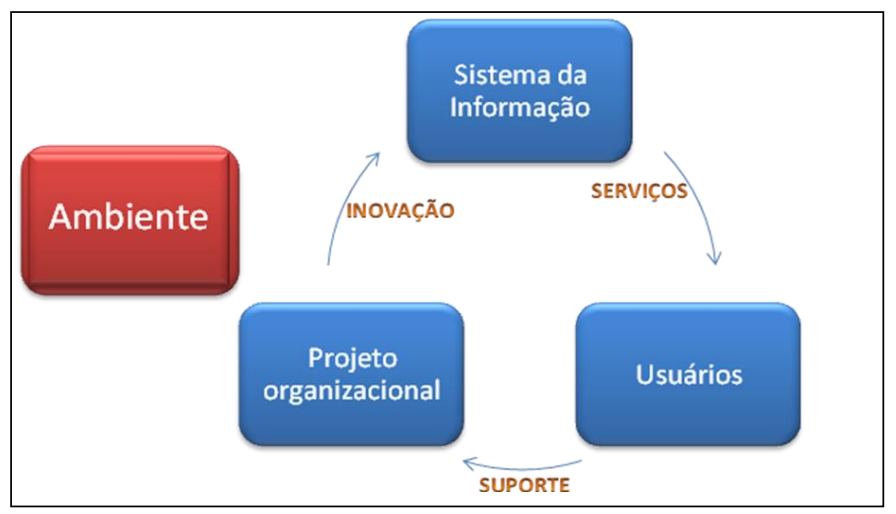

Fonte: Adaptada de Sauer (1993).

Sauer (1993) propôs que uma falha no sistema da informação (SI) deveria ser considerada apenas quando o desenvolvimento ou a operação pára ou os usuários ficam descontentes à medida que o sistema não corresponde aos seus interesses; ele desenvolveu um modelo baseado na insuficiência das interações entre os três grandes componentes: a) o projeto organizacional; b) o sistema informacional; e c) seus usuários.

Revista de Gestão e Projetos - GeP, São Paulo, v. 2, n. 1, p 52-68, jan./jun. 2011. 


\section{ESTUDO DE CASO}

A empresa do estudo de caso é do ramo de informática com 5 anos no mercado, presta serviço nas três principais vertentes da tecnologia em informática: treinamento, consultoria e desenvolvimento de softwares. É parceira da Microsoft e, por isso, todos os projetos elaborados têm o uso de suas ferramentas. Também possui projetos de tecnologia inovadora desenvolvidos com recursos da FINEP - Financiadora de Estudos e Projetos do Ministério da Ciência e Tecnologia e da FACEPE - Fundação de Amparo à Ciência e Tecnologia do Estado de Pernambuco.

Os gestores têm formação acadêmica focada em: engenharia da computação, sistema da informação e técnico de computação. Quanto à rotina, foi observado o acúmulo de várias funções nas áreas comerciais, de recursos humanos e financeiros.

Outro elemento analisado foi o treinamento dos colaboradores. Na empresa estudo de caso existe uma forte preocupação de alinhá-lo com sua cultura, a qual está voltada para a plataforma Microsoft, com outras práticas gerenciais existentes na organização e com especificidades do setor. Com relação ao estudo dos projetos, sua descrição está de acordo com o evento que é considerado ponto crítico e é definido como o ponto observável da alteração na evolução dos projetos.

\subsection{EVENTO 1 - GERAÇÃO DA IDEIA OU DO CONCEITO DA INOVAÇÃO (POSITIVO)}

O evento é gerado a partir da percepção da necessidade da inovação e pode surgir de várias fontes. A principal delas é o próprio conhecimento acumulado dos funcionários da empresa, muitos deles participam ou participaram de competições de estudantes em informática (como a ImagineCup e a Copa de Robôs). Nessa etapa, a ideia é amadurecida pela análise da oportunidade de implementar um novo produto no mercado, feito por meio do brainstorming entre os gestores da empresa e quem a idealizou. É verificado se há recursos humanos, financeiros, metodológicos (software), se há como comprar os componentes de hardware para a elaboração da inovação.

\subsection{EVENTO 2 - FORMAÇÃO DE UMA EQUIPE (POSITIVO)}

Nessa etapa, os gestores decidem formar uma equipe multifuncional em razão ao fechamento ou acerto do cronograma de trabalho e do orçamento. Cada equipe tem um líder recémnomeado que detém o conhecimento específico e as experiências de desenvolvimento de um 
software e hardware, ele é responsável por compreender as funcionalidades do novo sistema e repassar a situação de cada projeto para os demais gestores, porém isso não é feito por meio de relatórios mas, oralmente, em reuniões formais semanais elaboradas na empresa estudo de caso. Em geral, a média de tempo para o fechamento de todas as informações é de três meses.

\subsection{EVENTO 3 - PLANEJAMENTO - ELABORAÇÃO DO PLANO DE AÇÃO E ORÇAMENTO (NEUTRO)}

Os dados originados no Evento 1 são a base para elaborar o plano de ação e o orçamento da inovação.

\subsection{EVENTO 4 - ELABORAÇÃO DO PROTÓTIPO (NEGATIVO)}

Elaborou-se o protótipo, como uma representação fiel do produto, com todos os elementos e componentes técnicos, estruturais para fabricar a inovação, conforme os padrões de qualidade demandados pelo mercado e pelas normas de produto do setor. Porém, foi através da elaboração do protótipo que as dificuldades ocorreram. O processo de desenvolvimento do software era complexo porque envolveu vários sistemas e formatos de dados e, muitas vezes, havia falta de conhecimento dos desenvolvedores. Outra situação era que, por diversas razões, o sistema não funcionava corretamente, o projeto parava, por pelo menos duas semanas, para se estudar a solução do problema ocorrido. Por vários motivos, o resultado não foi o esperado e essa etapa foi considerada como negativa.

\subsection{EVENTO 5 - LANÇAMENTO DA INOVAÇÃO NO MERCADO (NEUTRO)}

A inovação passou a ser desenvolvida em maior número e foi inserida no mercado. No início desse evento, a produção foi feita com baixo volume para poder testar o impacto do novo produto no portfólio da empresa e sua atuação no ambiente concorrencial. Isso foi feito para melhorar ou ratificar o processo de fabricação, distribuição da empresa e dos fornecedores e a previsão de volume e lucro.

Revista de Gestão e Projetos - GeP, São Paulo, v. 2, n. 1, p 52-68, jan./jun. 2011. 


\section{RESULTADOS}

Constatou-se que pode haver falhas em qualquer evento de um projeto de inovação, isso acontece devido ao seu caráter inédito. No entanto, problemas entre os sistemas de informação e a equipe começaram a aparecer no Evento 4. Isso ocorreu devido à inadequação do protótipo aos padrões de qualidade e funcionalidade.

Nesse caso, o líder do projeto demorou para resolver o problema, já que o conhecimento de desenvolvimento de parte do software estava concentrado em uma pessoa e era visto como complexo para os outros membros da equipe. Observa-se no ambiente interno as dificuldades de reter e captar profissionais qualificados, em especial para cargos gerenciais, que requerem qualificações profissionais de maior nível cognitivo para a gestão administrativa e política da empresa.

Contudo, o líder e a equipe atendiam às expectativas para a implementação das ideias do produto. Havia a infraestrutura adequada para apoiar o projeto e a opinião sobre as possíveis melhorias e incrementos ao produto não foram ignorados.

Empresas inovadoras geralmente combinam diferentes fontes de inovação e conhecimento dos ambientes interno e externo. As fontes internas de inovação envolvem tanto as atividades explicitamente voltadas para o desenvolvimento de produtos e processos quanto à obtenção de melhorias incrementais por meio de programas de qualidade, seleção e treinamento de recursos humanos e aprendizado organizacional.

As fontes externas, segundo Bastos (2006), envolvem: a aquisição de informações codificadas, a exemplo de livros e revistas técnicas, manuais, software, vídeos; consultorias especializadas; obtenção de licenças de fabricação de produtos e tecnologias embutidas em máquinas ou equipamentos. Dessa forma, as ferramentas para a produção da inovação das fontes internas são elaboradas pelo uso da criatividade, conhecimento, expertise e qualificação dos próprios gestores e de seus colaboradores, como exemplo o emprego de metodologias de desenvolvimento ágil para o controle da produção da inovação e reuniões internas para acompanhar o andamento do projeto e o afloramento de sugestões. Para levantar as necessidades da demanda foi feita uma pesquisa de mercado em sites, jornais, revistas e fontes primárias como o IBGE, como está apresentado na Tabela 2.

Revista de Gestão e Projetos - GeP, São Paulo, v. 2, n. 1, p 52-68, jan./jun. 2011. 
Tabela 2 - Fatores que afetaram o desenvolvimento do projeto de inovação nas PMET.

\begin{tabular}{|c|c|c|c|c|c|}
\hline $\begin{array}{c}\text { SI } \\
\text { COMPONENTE } \\
\text { DE EXECUÇÃO }\end{array}$ & $\begin{array}{l}\text { EVENTO } 1 \\
\text { (POSITIVO) }\end{array}$ & $\begin{array}{c}\text { EVENTO } 2 \\
\text { (POSITIVO) }\end{array}$ & $\begin{array}{l}\text { EVENTO } 3 \\
\text { (NEUTRO) }\end{array}$ & $\begin{array}{c}\text { EVENTO } 4 \\
\text { (NEGATIVO) }\end{array}$ & $\begin{array}{l}\text { EVENTO } 5 \\
\text { (NEUTRO) }\end{array}$ \\
\hline SI & $\begin{array}{l}\text { - Análise da } \\
\text { ideia. }\end{array}$ & $\begin{array}{l}\text { - Forte } \\
\text { envolvimento } \\
\text { dos líderes. }\end{array}$ & $\begin{array}{l}\text { - Saber qual } \\
\text { metodologia irá } \\
\text { utilizar. } \\
\text { - Traçar plano } \\
\text { de trabalho. }\end{array}$ & $\begin{array}{l}\text { - Falha na } \\
\text { comunicação. }\end{array}$ & $\begin{array}{l}\text { - Melhorar o } \\
\text { produto } \\
\text { lançado. }\end{array}$ \\
\hline $\begin{array}{c}\text { Projeto } \\
\text { organizacional }\end{array}$ & $\begin{array}{l}\text { - Dilema da } \\
\text { seleção dos } \\
\text { recursos } \\
\text { (humanos e } \\
\text { financeiros, } \\
\text { matéria-prima). }\end{array}$ & $\begin{array}{l}\text { - Confiança na } \\
\text { relação líder e } \\
\text { equipe. }\end{array}$ & $\begin{array}{l}\text { - Forte } \\
\text { envolvimento } \\
\text { da equipe. } \\
\text { - Afirmação de } \\
\text { parcerias e } \\
\text { fontes de } \\
\text { financiamento. }\end{array}$ & $\begin{array}{l}\text { - Gestão de } \\
\text { pequenos } \\
\text { problemas } \\
\text { ignorados pelo } \\
\text { líder do projeto. }\end{array}$ & $\begin{array}{l}\text { - Iniciar outro } \\
\text { projeto } \\
\text { inovativo. }\end{array}$ \\
\hline Usuários & $\begin{array}{l}\text { - Levantamento } \\
\text { das } \\
\text { necessidades. }\end{array}$ & $\begin{array}{l}\text { - Levantamento } \\
\text { das } \\
\text { necessidades. }\end{array}$ & $\begin{array}{l}\text { - Contratação } \\
\text { de empresas } \\
\text { fornecedoras } \\
\text { dos } \\
\text { componentes. }\end{array}$ & $\begin{array}{l}\text { - Teste de } \\
\text { validação do } \\
\text { protótipo. } \\
\text { - Frustração da } \\
\text { equipe. }\end{array}$ & $\begin{array}{l}\text { - Espera pela } \\
\text { aceitação do } \\
\text { projeto. } \\
\text { - Moral alto da } \\
\text { equipe. }\end{array}$ \\
\hline
\end{tabular}

Fonte: Elaborado pelos autores.

Assim, percebe-se que os ativos intangíveis ou organizacionais de uma empresa estão reunidos para melhor atuar com o ambiente externo e promover a vantagem competitiva para a empresa (Yeoh e Roth, 1999).

O porte empresarial mantém o modelo gerencial adhocratico, com poucos níveis hierárquicos, o que eleva à flexibilidade e ao potencial de inovação (Gunesekaran et al., 2001), uma vez que estão mais próximas dos clientes (McAdam, 2000), e que permite o desenvolvimento de relações políticas mais cordiais, nas quais a gestão personalizada se torna uma característica de vantagem estratégica, percebe-se que há pouca incidência de falha na comunicação nos eventos.

Apenas nos Eventos 3 e 4 houve falhas no relacionamento entre os componentes: suporte e serviços na elaboração do protótipo, como estão demonstrados na Figura 2. Os erros são catalogados como histórico para a tomada de decisões futuras.

Revista de Gestão e Projetos - GeP, São Paulo, v. 2, n. 1, p 52-68, jan./jun. 2011. 
Figura 2- Diagrama PRISM da descrição do projeto de inovação da empresa estudo de caso, com base nos dados da Tabela 2.

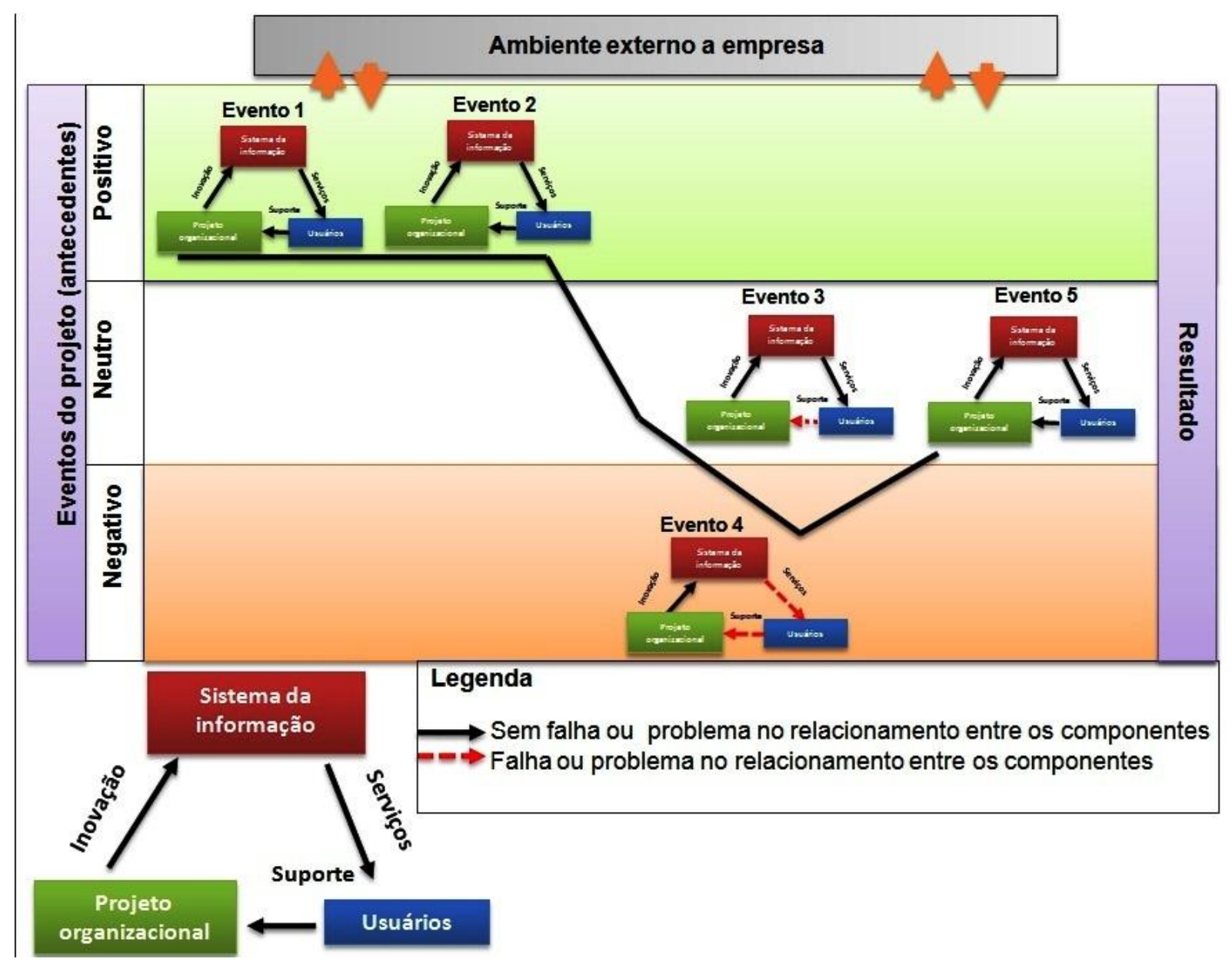

Fonte: Elaborado pelos autores.

Todos os projetos de inovação têm sua execução prevista dentro da tríade: tempo, custo e qualidade, desse modo, o fracasso do projeto é caracterizado pela incapacidade de implementar um conjunto de resultados dentro desses condicionalismos (Ewusi-Mensah, 1997). Frequentemente, são projetos de execução pontuada por resultados inesperados que requerem dos gerentes uma habilidade em desenvolver a ideia em protótipo (Ciborra, 2000). Em alguns casos, a execução requer adaptações ou reinvenções da solução inicial, esse fenômeno ocorre quando há maiores quantidades de informação.

Essa investigação revelou a importância de se estudar o caminho da elaboração do projeto de cunho inovativo e ver de modo não isolado, todas as partes que se relacionam para a tomada de decisão.

Revista de Gestão e Projetos - GeP, São Paulo, v. 2, n. 1, p 52-68, jan./jun. 2011. 


\section{CONSIDERAÇÕES FINAIS}

As empresas podem alcançar singularidade perante seus concorrentes pela inovação. Essa singularidade relaciona-se às pessoas, à gestão das competências de forma estratégica, ao controle e aos fatores que determinam o desempenho da empresa, sustentam sua vantagem competitiva e fornecem a dinamicidade capaz de antever as necessidades do mercado por meio da inovação.

Nesse sentido, o papel do líder é fundamental e refere-se à capacidade que o gerente tem de sensibilizar e associar o comprometimento de seus subordinados para com as estratégicas empresariais voltadas ao desenvolvimento de projetos inovativos.

A partir da reflexão sobre as particularidades da análise post-mortem de desenvolvimento de projetos em empresas de alta tecnologia, os obstáculos e desafios enfrentados pelas pequenas e médias empresas podem ser analisados no processo de inovação. Já que o próprio ciclo da inovação se confunde com a elaboração do projeto, no qual a própria geração de tecnologia se desenvolve por meio da transferência dos conhecimentos acumulados pelas dimensões: indivíduos, equipes e organizações.

\section{REFERÊNCIAS}

Bastos, T. P. (2006). Gestão da inovação: a economia da tecnologia no Brasil. Rio de Janeiro: Elvesier.

Campos, S. H. P. e Barbieri, J. C. (2002, novembro). Capital de risco para empresas de base tecnológica; uma avalização da atual legislação brasileira. Anais do XXII Simpósio de Gestão da Inovação Tecnológica, Salvador, BA, Brasil, 6-9.

Carvalho, M. M. e Rabechini, R. Jr. (2008). Construindo competências para gerenciar projetos (2a ed.). São Paulo: Atlas.

Castellano, S. (1996). Proposição de um modelo para planejamento e desenvolvimento de projetos em empresas de alta tecnologia. Dissertação de mestrado, Engenharia de Produção, Universidade Federal de Santa Catarina, Florianópolis, SP, Brasil. Recuperado em 20 janeiro, 2010, de http://www.eps.ufsc.br/disserta96/castellano/index/index.htm.

Revista de Gestão e Projetos - GeP, São Paulo, v. 2, n. 1, p 52-68, jan./jun. 2011. 
Ciborra, C. (2000). A critical review of the literature on the management of corporate information infrastructure. In C. Ciborra (Ed.). From control to drift. Oxford: Oxford University.

Drummond, H. (2005). What we never have, we never miss? Decision error and the risks of premature termination. Journal of Information Technology, 20(3), 170-178.

http://dx.doi.org/10.1057/palgrave.jit.2000046

Ewusi-Mensah, K. (1997). Critical issues in abandoned information systems development projects. Communications of the ACM, 40(9), 74-80.

http://dx.doi.org/10.1145/260750.260775

Fonseca, S. A. e Kruglianskas, I. (2002). Inovação em microempresas de setores tradicionais: estudos de casos em incubadoras brasileiras. In R. Sbragia e E. Stal (Eds.). Tecnologia e inovação: experiência de gestão na micro e pequena empresa (pp. 89-109). São Paulo: PGT/USP.

Freire, A. (2000). Inovação, novos produtos, serviços e negócios em Portugal. Lisboa: Verbo.

Gunesekaran, A.; Forker, L.; and Kobu, B. (2000). Improving operations performance in a small company: a international case study. Journal of Operations and Production Management, 20(3), 316-336.

http://dx.doi.org/10.1108/01443570010308077

IBGE - Instituto Brasileiro de Geografia e Estatística. (2005). Pesquisa de Inovação Tecnológica PINTEC 2005. Recuperado em 19 janeiro, 2009, de http:// www.ibge.gov.br/home/estatistica/economia/industria/pintec/2005/default.shtm.

IBGE - Instituto Brasileiro de Geografia e Estatística. (2006). Cadastro Central de Empresas CEMPRE 2006. Recuperado em 25 janeiro, 2009, de http:// www.ibge.gov.br/home/estatistica/economia/cadastroempresa/2006/.

Jerônimo, T.; Batista, D.; Staszczak, L. e Medeiros, D. (2009, outubro). Um estudo exploratório da construção de competências através das ferramentas de gestão em auxílio às PME de tecnologia de ponta. Anais do XXIX Encontro da Engenharia de Produção - Enegep, Salvador, BA, Brasil, 6-9.

Marcovitch, J.; Santos, S. e Dutra, I. (1986, abril/junho). Criação de empresas com tecnologias avançadas: as experiências do PACTo/IA-FEA- USP. Revista de Administração FEA/USP RAUSP, São Paulo, 21(2), 3-9.

McAdam, R. (2000). Quality models in a SME context. International Journal of Quality and Reliability Management, 17(3), 305-323. http://dx.doi.org/10.1108/02656710010306166

Revista de Gestão e Projetos - GeP, São Paulo, v. 2, n. 1, p 52-68, jan./jun. 2011. 
Murphy, A. and Ledwith, A. (2007). Project management tools and techniques in high-technology SMEs. Management Research News, 30(2), 153-66.

http://dx.doi.org/10.1108/01409170710722973

Organisation for Economic Co-operation and Development. (2000, June). Small and medium-sized enterprises: local strength, global reach. Policy Brief, OECD. Recuperado em 2 novembro, 2009, de http://www.oecd.org/dataoecd/3/30/1918307.pdf.

Pan, G.; Hackney, R.; and Pan, S. (2008). Information systems implementation failure: insights from prism. International Journal of Information Management, 28, 259-269.

http://dx.doi.org/10.1016/j.ijinfomgt.2007.07.001

PMBOK. (2004). A Guide to the Project Management Body of Knowledge (3a ed.). Newtown Square, PA, USA: Project Management Institute.

Sauer, C. (1993). Why information systems fail: a case study approach. Henley-on-Thames, Oxfordshire, UK: Waller.

Turner, J. R.; Ledwith A.; and Kelly, J. (2009). Project management in small to medium-sized enterprises: a comparison between firms by size and industry. International Journal of Managing Projects in Business, 2(2), 282 -296.

http://dx.doi.org/10.1108/17538370910949301

Vendrametto, O. (2007). Decisões em tecnologia. In P. L. O. Costa Neto (Coord.). Qualidade e competência nas decisões (1a ed.). São Paulo: Blucher.

Yeoh, P. and Roth, K. (1999). An empirical analysis of sustained advantage in the US pharmaceutical industry: impact of firm resources and capabilities. Strategic Management Journal, 20, 637-653.

http://dx.doi.org/10.1002/(SICI)1097-0266(199907)20:7<637::AID-SMJ42>3.3.CO;2-Q

http://dx.doi.org/10.1002/(SICI)1097-0266(199907)20:7<637::AID-SMJ42>3.0.CO;2-Z

Revista de Gestão e Projetos - GeP, São Paulo, v. 2, n. 1, p 52-68, jan./jun. 2011. 
Data do recebimento do artigo: 17/02/2011

Data do aceite de publicação: 30/04/2011

Revista de Gestão e Projetos - GeP, São Paulo, v. 2, n. 1, p 52-68, jan./jun. 2011. 